\title{
Anatomical and physiological patterns of right ventricle
}

\begin{abstract}
Until recently, only few studies have been devoted to the right ventricle. Moreover, this chamber was considered as just a passive conduit between the right atrium and the pulmonary artery. Only after increasing use of new non-invasive diagnostic techniques in last one-two decades made it possible to redefine physiological role of the right ventricle on maintenance of stabile central hemodynamics as well as its clinical importance in development of valvular diseases and other conditions associated with left ventricular dysfunction. This review summarizes anatomical-physiological characteristics of the right ventricle, its functional relation to the left heart within an overall interaction of two chambers with a special regard to the interventricular septum. Key patterns of the right ventricular blood supply are discussed.
\end{abstract}

Volume 2 Issue I - 2015

\author{
Hamlet Hayrapetyan \\ Department of Urgent Cardiology, Yerevan State Medical \\ University, Armenia
}

Correspondence: Hamlet Hayrapetyan, Department of Urgent Cardiology, Erebouni Medical Center,Titogradyan street 14, 0087 Yerevan, Armenia, Tel 3749|505005, Fax 37410473800, Email cardioerebouni@yahoo.com

Received: December 15, 2014 | Published: January 28, 2015

Keywords: right ventricle, left ventricle, geometry, preload, after load, blood supply

Abbreviations: SA, sino-atrial; LCX, left circumflex coronary artery; RCA, right coronary artery; PDA, posterior descending artery; LAD, left anterior descending artery; RVOT, right ventricular outflow tract; IVRT, isovolumic relaxation time; AV, atrioventricular; IVS, interventricular septum

\section{Introduction}

Anatomy of right ventricle (RV) and some of its function was first described by Harvay W. ${ }^{1}$ Since then, for many years until recent decades only a few researchers have tried to specify its prognostic importance in main cardiac diseases as right heart has been traditionally considered to have a little or at most secondary to left ventricle (LV) role in overall cardiac function and development of heart failure (HF).,3 Researchers underestimated RV physiological importance in the maintenance of normal overall hemodynamic performance and described this chamber as just a passive conduit with a little pumping capability which just connects right atrium (RA) with pulmonary artery. ${ }^{4}$ Such approaches were mainly due the fact that the evaluation of RV function was technically difficult (difficulty in accurately measuring RV volume as a result of its complex geometry etc.).

Introduction and common use of modern non-invasive diagnostic methods and techniques (first of all echocardiography and magnetic resonance imaging) in cardiology since 1990s have allowed imagebased quantitative assessment of ventricular mechanics. ${ }^{5,6}$ Importance of RV was recognized after some diagnostic criteria have been defined to evaluate functions of both ventricles. ${ }^{7}$ Over the last decades, numerous studies have demonstrated the physiological and clinical importance of RV function. Currently, the role of RV function in many conditions and valvular diseases are not in question. ${ }^{8-10}$ Today RV function is redefined and this ventricle used to be a "forgotten" camber is seen as one which has unique anatomical patterns and significant physiological importance on one hand, and RV dysfunction is considered an important predictor of overall survival and morbidity in different clinical settings on the other hand. ${ }^{11,12}$

\section{Anatomy and physiology of right ventricle}

Functional imaging capabilities are improved and it is now well appreciated that there exists some independence between the LV and RV systolic function and diastolic load. ${ }^{13}$ Current researchers show an increasing interest in the RV particularly with regard to RV dysfunction and RV HF associated with ST-elevation myocardial infarction with an involvement of the same ventricle. For this reason as well as to boost further studies in the field of RV physiology, in 2006, the National Heart, Lung, and Blood Institute defined RV function and RV HF as a priority in cardiovascular research. ${ }^{14} \mathrm{RV}$ muscle mass is about one-sixth that of the LV. This could be explained by different loading conditions of ventricles. ${ }^{15}$ While the normal LV is thick walled and ellipsoid in shape, the RV is thin walled (3-4mm) and complex shaped due to the interventricular septum (IVS).${ }^{16}$ Being an anatomical part of the LV, however, IVS is directed obliquely backward to the right, and curved with the convexity toward the RV. ${ }^{12}$

While the LV is conical shaped, the RV is more triangular and/or semi lunar in form on lateral and/or vertical projections. The shape of the RV is related to the IVS position. In addition, the RV differs from the LV by having strict trabeculae, septomarginal trabecula (or moderator band) and inter-valve fibros tissues. ${ }^{17}$ Contrary to the LV, the RV has much complex geometry. It consists of an inlet portion, and outlet portion and an apical trabecula portion between them. Some researchers consider such anatomical-physiological description of the RV much appropriate than traditional division into sinus and conus, because apical trabeculae allows distinguishing between morphologically right and left ventricles regardless of location of major mass of ventricle (Figure 1). Muscular trabeculae are thicker in the apex of morphological RV while they are smooth and crosswise within the LV. ${ }^{17-19}$

Under normal conditions, effective stroke volumes of both ventricles are almost the same. ${ }^{20}$ However, under the same rate and stroke volume the LV and RV differs significantly in the oxygen delivered by coronary blood system - about $75 \%$ and $50 \%$ correspondingly, mainly due to the low pulmonary resistance to keep central venous pressures low and prevent organs and tissues from congested impairment. ${ }^{14,21}$ Related to the abovementioned, the RV generates significantly lower pressures with shorter RV isovolumic contraction time (IVCT). ${ }^{22}$ The IVS and free wall have almost equal contributions to the RV performance.

While the LV consists of supepicardial oblique, subendocardial longitudinal and medial circular myofibers, the RV free wall is mainly composed of circumferentially oriented and parallel to the 
atrioventricular (AV) groove in subepicardium and longitudinally arranged from base to apex deep muscle layers in subendocardium so that such a structure ensures the more complex movement of the RV - torsion, translation, rotation, and thickening. ${ }^{17,21}$ In general, longitudinal contraction is a greater contributor to the RV effective stroke volume than circumferential one. ${ }^{22}$

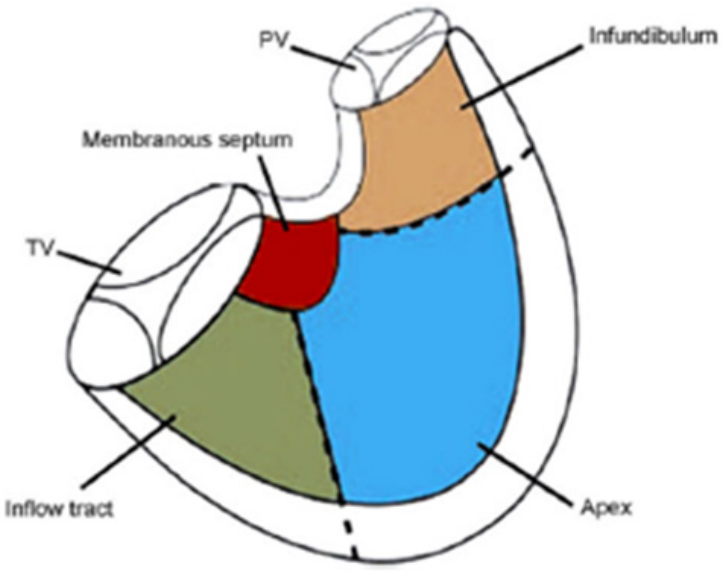

Figure I Diagram of the right ventricle demonstrating its 3 major chamber components; inflow tract, infundibulum (outflow tract), and apex (Adopted from Kenneth et al., 2009)

The RV shortening is sequential. It starts with the contraction of the inlet and trabeculated segments and ends with the contraction of the infundibulum or the conus (about 25-50ms apart). Contraction of the outflow region lasts longer than that of the inflow region. In general, the RV contracts much in longitudinal rather than radial way. ${ }^{23}$ Left-to-right systolic ventricular interaction arises from intraventricular transmission of systolic forces through the IVS and from the mechanical effect of the common muscle fibers encircling both chambers. ${ }^{24,25}$ Most importantly, any drop in LV free wall function immediately brings to a lower RV function. ${ }^{26}$ Mutually systolic interaction between the LV and RV mainly works through the IVS yet pericardium has a little role. Rather, the pericardium becomes important in diastolic interaction of two ventricles. Systolic ventricular interaction is more important for RV systolic function, and one - third of the RV potential systolic pressure is generated by the LV.

Conversely, the RV pressure overload causes significant disturbance of LV function and in some cases may lead to acute LV HF. Similarly, in cases when LV HF is associated with the RV failure, the RV may become unable to supply the effective flow volume for maintaining adequate LV preload. In such and other cases, RV failure may lead to a "common final pathway" resulting in the progression of congestive HF. Thus, in LV HF settings RV dysfunction can be considered as a unique indicator of bad prognosis. ${ }^{27}$ The primary function of the RV is to facilitate blood flow through the lung. Today it is commonly accepted that the normal function of the RV is depending upon the complex relationship between contractility, preload, and after load of that chamber. Such mutual relationships can be better described with the help of pressure vs. volume loop analysis in terms of pressure-volume curves under different loading conditions. In such analyses a ventricular elastance (the slope of the end-systolic pressure-volume relationship) stands as the most reliable index of ventricular contractility. ${ }^{28}$

In general, the RV after load integrates resistance, elastance, and wave reflection. ${ }^{29}$ In contrary to the $\mathrm{LV}$, the RV is more sensitive to after load change. In clinical settings, pulmonary vascular resistance is the most commonly used measurement of after load. On the other hand, as the RV preload increases, performance of this chamber also increases according to the Frank-Starling mechanism. However, beyond the physiological range abovementioned mechanism stops working and excessive RV volume loading causes the LV dysfunction through the mechanism of ventricular interdependence. ${ }^{30}$ Compared with the LV filling, the RV filling normally starts earlier and finishes later. At the same time, the RV isovolumic relaxation time (IVRT) is shorter and RV filling velocities are lower. The respiratory variations in RV filling velocities are more pronounced. There are many other factors influencing filling of the RV such as ventricular chamber compliance, heart rate, ventricular relaxation, etc., ${ }^{31}$ The RV follows a force-interval relationship, that is, when filling period's last longer stroke volume increases above baseline. In addition, the RV is more compliant than the LV. ${ }^{32}$ The pericardium imposes greater constraint on the thin walled and more compliant RV. ${ }^{19}$ Acute RV ischemia is shown to bring to significant shifts in IVCT and partially IVRT. ${ }^{27}$

\section{Blood supply of the right ventricle}

The RV has its unique blood supply system. It is a part of the overall coronary circulation consisting of coronary arteries arising from the ascending aorta. The coronary arteries run along the outside of the heart. They have small branches that dive into the heart muscle to bring it blood. Two main coronary arteries are left coronary artery (also known as the left main) (LCA) and right coronary artery (RCA). The LCA supplies blood to the left side of the heart muscle - the left atrium, IVS, LV and the anterior wall of the RA. On the other hand, the RCA supplies blood to the right side of the heart muscle - the RA, the RV and the Sino-atrial (SA) node which regulate the heart rhythm. ${ }^{33}$

The RV receives arterial blood through the RCA as well as two branches of the LCA - left anterior descending artery (LAD) and left circumflex coronary artery (LCX). Starting from the right aortic sinus, the RCA runs towards the right side of the heart, then deeps to the $\mathrm{RA}$ along the right $\mathrm{AV}$ groove and curls around towards the inferior surface of the heart. In $90 \%$ of patients this artery forms the posterior interventricular branch at the crux of the heart, more commonly known as the posterior descending artery (PDA). The distal extent of the RCA may extend posteriorly reaching the left margin of the heart. Running along the posterior interventiculas sulcus to the apex of the heart the PDA provides blood supply of the LA and RV walls, $\mathrm{AV}$ node and the posterior parts of the IVS (Figure 2). The first (the conal or infundibular) branch of the RCA runs anteriorly and provides blood supply of the muscular right ventricular outflow tract (RVOT) or infundibulum. The RCA supplies blood to the atria with small blood vessels. In approximately $50 \%$ of patients the proximal RCA gives rise of the sinus node artery. Originating from the single short ostium, a short, common LCA truck branches into the LAD and the LCX. Running in the anterior interventricular groove, the LAD gives anterior septal perforating branches to supply blood to the cardiac apex. Other small branches of the LAD supply the anterior wall of the $\mathrm{RV}$ and diagonal branches supply the anterolateral free wall of the LV.

The LCX courses within the posterior (left AV) groove, around the obtuse margin, and posteriorly toward the crux of the heart. In about $10 \%$ of patients the LCX reaches the crux of the heart and supply the PDA (left domination). Atrial branches may arise from the LCX and supply the sinus node in $40 \%$ of patients. One to three large obtuse marginal branches arise from the circumflex system to supply the lateral free wall of the LV. In an estimated $70 \%$ of patients, the intermedius branch arises early of the left coronary system to supply an area between diagonal branches from the LAD and obtuse 
branches from the circumflex systems. Before turning towards the diaphragmatic surface of the heart the RCA gives rise to the right marginal branch which runs along the right margin, to supply the wall of the RV. Again, the RCA gives rise to several more branches such as the conus branch and the SA node artery, passing upwards to the aurical wall to the junction between superior vena cava, sulcus terminals and right auricle.

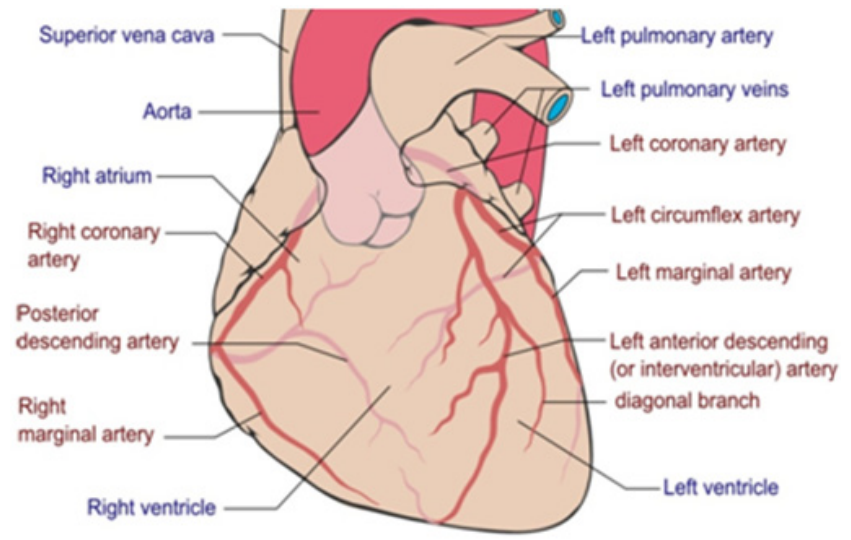

Figure 2 Coronary artery system.

In less than $1 \%$ of the general population some variations of abovementioned coronary supply system may be found, but most of such anomalies have little or no clinical importance. In exceptional cases a high origin of the ostia may reduce diastolic coronary artery blood flow. Supply of the RV differs according to the dominance of the coronary system which is determined by the artery that supplies the PDA. First, if the PDA is supplied by the RCA, then the coronary circulation is termed "right-dominant". Second, when the PDA is supplied by the LCX (a branch of the left artery), then the coronary circulation is termed "left-dominant". Finally, the coronary circulation is termed "co-dominant" if the PDA is supplied by both the RCA and the LCX. ${ }^{34}$ The right-dominant system is found in about $80 \%$ of the population. Here the RCA supplies most of the RV.. ${ }^{21,34}$ The lateral wall of the RV receives blood through the marginal branches of the $\mathrm{RV}$, whereas the posterior wall and the inferoseptal region - by the PDA. The anterior wall of the RV and the anteroseptal region receives blood through branches of the left anterior descending artery while the infundibulum is supplied by the conal artery, which has a separate ostial origin in $30 \%$ of cases. The separate ostium explains the preservation of infundibular contraction in the presence of proximal right coronary occlusion. ${ }^{21}$

\section{Conclusion}

In normal individuals, proximal RCA perfusion occurs during both systole and diastole. However, beyond the RV marginal branches, diastolic coronary blood flow predominates. The relative resistance of the RV to irreversible ischemic injury may be explained by its lower oxygen consumption, its more extensive collateral system, especially from the moderator band artery, a branch of the first septal perforator that originates from the left anterior descending coronary artery, and its ability to increase oxygen extraction. ${ }^{35}$

\section{Acknowledgments}

None.

\section{Conflicts of interest}

Author declares there are no conflicts of interest.

\section{Funding}

None.

\section{References}

1. Harvey W. Exercitatio Anatomica de Motu Cordis et Sanguinis in Animalibus. Prometheus Books, New York, USA. 1628.

2. Goldstein J. The right ventricle: what's right and what's wrong. Coron Artery Dis. 2005;16:1-3.

3. Zaffran S, Kelly R, Meilhac S, et al. Right ventricular myocardium derives from the anterior heart field. Circ Res. 2004; 95(3): 261-268.

4. Rigolin V, Robiolio P, Wilson J, et al. The forgotten chamber: the importance of the right ventricle. Cathet Cardiovasc Diagn. 1995;35(1):18-28.

5. Badano L. The EAE Textbook of Echocardiography. European Society of Cardiology, Oxford University Press, Oxford, UK. 2011.

6. Tourneau T, Piriou N, Donal E, et al. Imaging and modern assessment of the right ventricle. Minerva Cardioangiol. 59(4): 349-373.

7. Bleasdale R, Frenneaux M. Prognostic importance of right ventricular dysfunction. Heart. 2002; 88(4):323-324

8. Noordegraa A, Galie N. The role of the right ventricle in pulmonary arterial hypertension. Eur Respir Rev. 2011;20(122):243-253.

9. Sallach J, Tang W, Borowski A, et al. Right atrial volume index in chronic systolic heart failure and prognosis. JACC Cardiovasc Imaging. 2009;2(5):527-534.

10. Vecchia L, Zanolla L, Varotto L, et al. Reduced right ventricular ejection fraction as a marker for idiopathic dilated cardiomyopathy compared with ischemic left ventricular dysfunction. Am Heart J. 2001;142(1):181-189.

11. Moazami N, Hill L. Right ventricular dysfunction in patients with acute inferior MI: role of RV mechanical support. Thorac Cardiovasc Surg. 2003;51(5):290-292.

12. Dell'Italia L. Anatomy and Physiology of the Right Ventricle. Cardiol Clin. 2012;30(2):167-187.

13. Hsu S, Lin J, Chang S. Right ventricular function in patients with different infarction sites after a first acute myocardial infarction. Am J Med Sci. 2011;342(6):474-479.

14. Voelkel N, Quaife R, Leinwand L, et al. Right Ventricular Function and Failure: Report of a National Heart, Lung, and Blood Institute Working Group on Cellular and Molecular Mechanisms of Right Heart Failure. Circulation. 2006;114(17):1883-1891.

15. Pinsky M, Teboul JL. Assessment of indices of preload and volume responsiveness. Curr Opin Crit Care. 2005;11(3):235-239.

16. Foale R, Nihoyannopoulos P, McKenna W, et al. Echocardiographic measurement of the normal adult right ventricle. Br Heart $J$. 1996;56(1):33-44.

17. Ho SY, Nihoyannopoulos P. Anatomy, echocardiography, and normal right ventricular dimensions. Heart. 2006;92 Suppl1:i2-i13.

18. Goor D, Lillehei C. Congenital malformations of the heart. (1st edn) In: Grune et al. (Eds.), New York, USA. 1975. p.1-37.

19. Lindqvist $P$, Calcutteea A, Henein M. Echocardiography in the assessment of right heart function. Eur J Echocardiogr. 2008;9(2):225-234.

20. Haddad F, Hunt S, Rosenthal D, et al. Right ventricular function in cardiovascular disease, part I: anatomy, physiology, aging, and functional assessment of the right ventricle. Circulation . 2008;117(11):1436-1448.

21. Dell'Italia LJ. The right ventricle: anatomy, physiology, and clinical importance. Curr Probl Cardiol. 1991;16(10):653-720. 
22. Kukulski T, Hubbert L, Arnold M, et al. Normal regional right ventricular function and its change with age: a Doppler myocardial imaging study. $J$ Am Soc Echocardiogr. 2000;13(3):194-204.

23. Davidson C, Bonow R. Cardiac catheterization. In: Zipes D, et al. (Eds.), Braunwald's Heart Disease: A Textbook of Cardiovascular Medicine. (7th edn), Elsevier, Philadelphia, USA. 2005.

24. Lumens J, Arts T, Marcus J, et al.Early-Diastolic Left Ventricular Lengthening Implies Pulmonary Hypertension-Induced Right Ventricular Decompensation. Cardiovasc Res. 2012;96(2):286-295.

25. Osculati G, Malfatto G, Chianca R, et al. Left-to-right systolic ventricular interaction in patients undergoing biventricular stimulation for dilated cardiomyopathy. J Appl Physiol . 2010;109(2):418-423.

26. Jerzewski A, Steendijk P, Pattynama PM, et al. Right ventricular systolic function and ventricular interaction during acute embolisation of the left anterior descending coronary artery in sheep. Cardiovasc Res. 1999;43(1):86-95

27. Simon M, Pinsky MR. Right ventricular dysfunction and failure in chronic pressure overload. Cardiol Res Pract. 2011;2011:568095.

28. Starling M, Walsh R, Dell'Italia L, et al. The relationship of various measures of end-systole to left ventricular maximum time-varying elastance in man. Circulation. 1987;76(1):32-43.
29. Naeije R. Pulmonary vascular function. In: Peacock AJ et al. (Eds.), Pulmonary Circulation. Arnold, London, UK. 2008. p.3-11.

30. Chin K, Kim N, Rubin L. The right ventricle in pulmonary hypertension. Coron Artery Dis. 2005; 16(1):13-18.

31. Burgess M, Mogulkoc N, Bright-Thomas R, et al. Comparison of echocardiographic markers of right ventricular function in determining prognosis in chronic pulmonary disease. J Am Soc Echocardiogr. 2002; 15(6): 633-639.

32. Gaasch W, Cole J, Quinones M, et al. Dynamic determinants of left ventricular diastolic pressure-volume relations in man. Circulation. 1975;51(2):317-323

33. Smithuis R, Willems T. Coronary anatomy and anomalies. The Radiology Assistant. 2008.

34. Fuster V, Alexander R, O'Rourke R. Hurst>s The Heart. (10th edn), McGraw-Hill, New York, USA. 2000. p.53.

35. Janik M, Chappell C, Green T, et al. Two coincident coronary anomalies: absent left main coronary artery and origin of the right coronary artery from the middle left anterior descending artery. Tex Heart Inst J. 2009;36(2):180-181. 